\title{
Fault Estimation and Accommodation for Virtual Sensor Bias Fault in Image-Based Visual Servoing using Particle Filter
}

\author{
Mien Van, Shuzi Sam Ge, Fellow, IEEE, Dariusz Ceglarek, Senior Member, IEEE
}

\begin{abstract}
This study develops a fault estimation and accommodation scheme for the image-based visual servoing (IBVS) system to eliminate the effects of the faults due to the image feature extraction task, which is named as bias virtual sensor fault. First, a bias virtual sensor fault in visual servoing is declared. Then, fault diagnosis (FD), which includes fault detection, isolation and estimation, is designed based on the means of particle filter (PF). Finally, a fault accommodation law is developed based on the information obtained from the fault estimation to compensate for the effects of the fault in the system. The proposed fault estimation and accommodation is verified through simulation and experimental studies, and the results show that the system can estimate and eliminate the unknown fault effects effectively.
\end{abstract}

Index Terms - Fault diagnosis, Fault tolerant control, Imagebased visual servoing, Particle filter, Robot control.

\section{INTRODUCTION}

厂 MAGE BASED VISUAL SERVOING (IBVS) has been proven as an effective method for the robotic system guided by visual information due to its easy in implementation and high accuracy [1]. There are increasing numbers of applications which can significantly benefit by using the IBVS approaches to extract position of the geometric feature/target which has inherent error. For example, robotic laser welding process guided by real-time seam tracking or edge detection (welding on the fly) [2]; or application of laser or white light scanners used for in-process or in-line 3D parts geometry inspections [3]. However, the current visual servoing approaches encounter some limitations as discussed below. In traditional visual servoing approaches, the image features are defined based on the geometric characteristic of the object such as

Manuscript received Aug 24, 2016; revised Dec 08, 2016, Feb 18, 2017 and May 04, 2017; accepted June 04, 2017. This work was partly in collaboration with Computational Intelligent and Applications Research Group at Nottingham Trent University. (Corresponding author: Mien Van).

Mien Van is with the Science and Technology, Nottingham Tren University, Nottingham, United Kingdom (e-mail: mien.van@ntu.ac.uk, vanmien1@gmail.com), Orcid ID: 0000-0001-9616-6061.

S. S. Ge is with the Department of Electrical and Computer Engineering and Social Robotics Laboratory, National University of Singapore, 117580, Singapore (e-mail: samge@nus.edu.sg).

Dariusz Ceglarek is with the WMG, University of Warwick, CV47AL, United Kingdom (e-mail: D. J. Ceglarek@warwick.ac.uk). points, ellipses, straight lines, or segments, etc [4-5]. The control law is calculated based on the displacement of the designed image features during visual servoing. Based on this principle, the robot tracks the object precisely when the displacements of all the designed image features are precisely identified. However, this major task has sometime been failed due to the effects of the complex environment during visual servoing [5-6]. In general, the failures can be caused by: (1) image singularities [6-7], (2) inadequate field of view (FOV) of camera [8-9]: due to the visibility constraint of the camera, some features may go out of FOV of camera during visual servoing, and (3) the environment noises: due to the change of the environment such as light condition, obstacles during visual servoing, some designed image features could be occluded, or some undesired image features could be appearing. In order to discard the image singularities, effective visual features such as polar features [6] or moment [7] have been proposed. To avoid the loss of features due to the visibility constraint, numerous published literatures have been developed to increase the FOV of camera [8-11]. The innovations of these methods have been thoroughly reviewed in [12]. To reduce the collision with obstacle, surface laser scanning has been developed [13]. Although these approaches can effectively avoid the failures due to the lack of FOV of camera, collision with obstacle and image singularities, the failure of the image feature extraction task due to the image noises has not been considered yet; in fact this failure scenario is usually occurred in real applications. For example, the bias fault due to image noise in seam extraction of robotic welding system as illustrated in Fig. 1: the demand of the feature extraction task in seam extraction for $\mathrm{V}$-groove type is to identify the three image feature points, as shown in Fig. 1a); however, due to the similar property of the desired feature point and the noise feature point, the system extract the noise image feature point instead of the desired feature point, and thus the displacement of the designed feature will be calculated incorrectly, as shown in Fig. 1b). In order to monitor the failure due to the feature extraction task, fault diagnosis observer based on Kalman filter has been developed $[12,14]$. In this approach, a nonlinear dynamic model of the visual servoing, in which the camera velocity is defined as the input and the displacements of the feature points are defined as the output, was investigated. Based on the defined dynamic 
model of the visual servoing, the failure of the feature extraction tasks can be considered as the virtual sensor faults $[12,14]$. Then, a fault detection and isolation scheme was established based on the Kalman filter. However, this approach has not yet considered the fault estimation, which is a crucial task to identify the severity level of the fault. In addition, the approach has not yet investigated for the fault accommodation, which is extremely desired in real applications to compensate for the effect of the fault to guarantee that the system can guarantee the desired performance even in the presence of fault.

In this paper, as a second part of our previous approach [12], we investigate a fault diagnosis scheme, which includes fault detection, isolation and estimation, for IBVS. The fault diagnosis observer is designed based on Particle filter (PF). The PF is employed because it has a good capability to handle nonlinear and non-Gaussian models, as well as it is robust and flexible compared to Kalman filter or other filters [15-18]. After a fault is diagnosed, it is desired that the controller should be reconfigured to reduce the effect of the fault [1920]. This task is known as fault accommodation, or fault tolerant control (FTC). Generally, there are two ways to compensate for the effects of a fault in the system [21]: (1) Passively, FTC is designed based on the assumption that the set of possible system faults can be predicted in advance, and a fixed control law is designed based on the predicted fault for both normal and fault operations [22]. However, the prior knowledge of the possible system fault is difficult to be obtained in visual servoing system since the level of noise of the system is difficult to get in advance. (2) Actively, namely active FTC (AFTC), the control law is adjusted based on the fault information, which is obtained from a fault diagnosis observer scheme [23-24]. The operation of the AFTC consists of two stages. In the first stage, a FD observer is designed to estimate the system faults online. In the second stage, the system uses the obtained fault information to reconfigure the control law. Compared to the passive FTC, the active approach has a better performance when the magnitude of fault is correctly estimated, and thus is desired in real applications.

In summary, the contribution of this paper can be marked by the following significant points:

- Virtual sensor fault in visual servoing system is broadly reviewed.

- A FD observer is designed to detect, isolate, and estimate the severity of virtual sensor fault, based on Particle filter.

- An AFTC control law is developed to compensate the effect of faults in the system.

The rest of this paper is organized as follows. In section II, problem formulation is stated. In section III, FD and FTC strategies based on Particle filter are presented. In section IV, we verify the proposed methodologies based on simulation study. The performance of the proposed strategies is further verified through experimental study detailed in section $\mathrm{V}$. The conclusion and future work are provided in section VI.

\section{II.PROBLEM FORMULATION}

Considering the pinhole perspective model of camera used in visual servoing system $[1,4]$, the projection of the $3 \mathrm{D}$ points $P_{i}=\left[x_{i}, y_{i}, z_{i}\right]^{T}, i=1, \ldots, n$ into the image plane of the camera is $s_{i}=\left[u_{i}, v_{i}\right]^{T}, i=1, \ldots, n$, where [12]:

$s_{i}=\left[\begin{array}{l}u_{i} \\ v_{i}\end{array}\right]=\frac{\lambda}{z_{i}}\left[\begin{array}{l}x_{i} \\ y_{i}\end{array}\right]$

where $\lambda$ is the focal length of the camera. The relationship between the displacement of the feature point in the image plane $\dot{s}$ and the spatial velocity of camera $V_{c}=\left[v_{x}, v_{y}, v_{z}, \gamma_{x}, \gamma_{y}, \gamma_{z}\right]^{T}$ can be expressed as:

$\dot{s}_{i}=L_{i}\left(s_{i}, z_{i}\right) V_{c}, i=1, \ldots, n$

where, $L_{i}\left(s_{i}, z_{i}\right)$ is the interaction matrix, and

$L_{i}\left(s_{i}, z_{i}\right)=\left[\begin{array}{cccccc}-\frac{\lambda}{z_{i}} & 0 & \frac{\lambda u_{i}}{z_{i}} & u_{i} v_{i} & -\left(1+u_{i}^{2}\right) & v_{i} \\ 0 & -\frac{\lambda}{z_{i}} & \frac{\lambda v_{i}}{z_{i}} & \left(1+v_{i}^{2}\right) & -u_{i} v_{i} & -u_{i}\end{array}\right]$

$z$ is the depth of the image features and is assumed to be known [4].

The target of the control law in visual servoing system is to minimize the error $\mathrm{e}$, which is defined as the different between the current feature point $s$ and their goal value $s^{*}$, $\mathrm{e}=s-s^{*}$. The traditional control law is designed as

$V_{c}=-\delta \hat{\mathrm{L}}_{\mathrm{s}}^{+} \mathrm{e}$

where $\delta$ is a positive gain and $\hat{\mathrm{L}}_{\mathrm{s}}^{+}$is an approximation of pseudoinverse of $\mathrm{L}_{s}=\left[L_{1}, L_{2}, . . L_{n}\right]^{T}$.

Discretization is applied to (2) [12]:

$\chi_{\mathrm{k}+1}=\mathrm{A} \chi_{\mathrm{k}}+\mathrm{B}_{k} v_{k}+\Delta_{k}+\omega_{k}$

$\psi_{k}=\mathrm{C} \chi_{k}+\zeta_{k}$

where $\chi=s=\left[u_{1}, v_{1}, \ldots, u_{n}, v_{n}\right]^{T}$ denotes the state variable of the system, $v=K V_{c}$ is the control input, where $K$ is the sampling time, $\Delta_{k}$ is used to represent the system uncertainty, which is defined as the error when discretizing (2) to (4) [12], $\omega$ and $\zeta$ are the model uncertainty and measurement noise, respectively. The coefficient matrix are defined as

$$
\begin{aligned}
& \mathrm{A}=\mathrm{I}_{(2 \times \mathrm{n}, 2 \times \mathrm{n})}, \mathrm{B}=L_{s}=\left[L_{1}, L_{2} \ldots, L_{n}\right]_{(2 \times \mathrm{n}, 6)}^{T} \\
& \mathrm{C}=\mathrm{I}_{(2 \times \mathrm{n}, 2 \times \mathrm{n})}
\end{aligned}
$$

When a virtual sensor fault occurs, the true value of the designed image feature may not be determined. This means that the input signal is measured as $\bar{s}(t)=s(t)+\Delta s(t)$, where

$s(t)$ is the true signal and $\Delta s(t)$ is the fault signal.

In the presence of a virtual sensor bias fault, the system dynamics is changed to

$$
\begin{aligned}
& \chi_{\mathrm{k}+1}=\mathrm{A} \chi_{\mathrm{k}}+\mathrm{B}_{k} v_{k}+\Delta_{k}+\omega_{k} \\
& \psi_{k}=\mathrm{C}\left(\chi_{k}+\theta(t)\right)+\zeta_{k}
\end{aligned}
$$




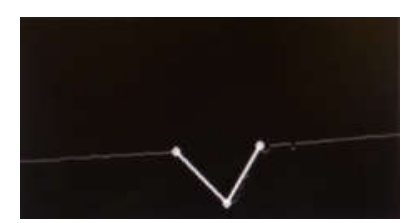

a)

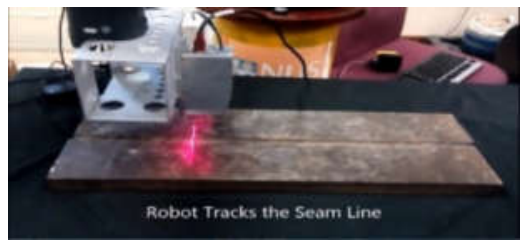

c)

Fig. 1. Sensor bias fault in weld seam extraction of a welding robot system. a) normal operation, b) virtual sensor bias fault, c) welding robot system. [12].

where the fault function is defined by

$$
\theta(t)= \begin{cases}0, & t \neq T_{s} \\ \Delta s(t), & t=T_{s}\end{cases}
$$

where $T_{S}$ is the time that the fault occurs.

The objective of this paper is twofold: (1) design a fault diagnosis scheme based on Particle filter to detect, isolate, and estimate the unknown fault $\theta(t)$, and (2) design a fault accommodation scheme such that the visual servoing can selfcompensate the effect of faults and continue to work reliably with an acceptable performance even though the faults still exist in the system.

\section{FAUlt Diagnosis AND FAUlt TOLERANT CONTROL BASED ON PARTICLE FILTER}

Based on the property of the faults, fault diagnosis problem can be categorized into three major tasks [19-21]: 1) Fault detection: makes a binary decision whether and when any abnormal event in the monitored system happens, or if everything works well, 2) Fault isolation: identifies the root of the fault, 3) Fault identification or fault estimation: specifies the magnitude of the fault. In the following the Particle filterbased fault diagnosis is presented.

\section{A. Particle Filter}

Consider the dynamic system of interest is described by

$$
\begin{aligned}
& \chi_{k}=f\left(\chi_{k-1}, \xi_{k-1}\right) \\
& \psi_{k}=h\left(\chi_{k}, \zeta_{k}\right)
\end{aligned}
$$

where $\chi_{k}$ is the state variable, $\psi_{k}$ is the measurement. The system and measurement noises $\xi_{k}$ and $\zeta_{k}$ are assumed to be independent of $\psi_{k}$. However, unlike the Kalman filter, they need not be Gaussian distributed. Since the visual servoing system described in (4) may not be a Gaussian distributed system due to the uncertainty $\Delta$, the Particle filter would be effective to approximate the system states.

From a Bayesian perspective, the problem of the state estimation is to calculate the probability density function (pdf) $p\left(\chi_{k} \mid \psi_{1: k}\right)$ of the state $\chi_{k}$ based on the sensor data available up to time $k, \psi_{1: k}=\left\{\psi_{1}, \psi_{2}, \ldots, \psi_{k}\right\}$. Starting from the values of the initial condition $p\left(\chi_{0} \mid \psi_{0}\right) \equiv p\left(\chi_{0}\right)$ and the pdf, $p\left(\chi_{k-1} \mid \psi_{1: k-1}\right)$ at time $k-1$, there are two steps to update the pdf at the time $k, p\left(\chi_{k} \mid \psi_{1: k}\right)$.

i) Prediction step:

$p\left(\chi_{k} \mid \psi_{1: k-1}\right)=\int p\left(\chi_{k} \mid \chi_{k-1}\right) p\left(\chi_{k-1} \mid \psi_{1: k-1}\right) d \chi_{k-1}$

ii) Update step:

$p\left(\chi_{k} \mid \psi_{1: k}\right)=\frac{p\left(\psi_{k} \mid \chi_{k}\right) p\left(\chi_{k} \mid \psi_{1: k-1}\right)}{p\left(\psi_{k} \mid \psi_{1: k-1}\right)}$

where $p\left(\psi_{k} \mid \psi_{1: k-1}\right)$ is a normalizing factor that depends on the pdf $p\left(\psi_{k} \mid \chi_{k}\right)$.

In theory, the Bayesian filter can estimate the true state variable by using the two above recursive steps (10) and (11). However, the approach can only give optimal solution if the system can satisfy two assumptions: the noises are Gaussian distribution and the system is linear. However, these assumptions are not usually satisfied in real applications. To overcome the limitation, Particle filter, which is an approximation method of Bayesian filter, has been proposed. The PF approximates the pdf using a set of $N$ particles, $\left\{\chi_{k}^{i}, \rho_{k}^{i}\right\}$, where $\chi_{k}^{i}$ presents the $i$ th particle and $\rho_{k}^{i}$ presents its associated weights.

In literature, many algorithms have been developed for the Particle filter. In this paper, we use sequential importance resampling (SIR) [15] due to its efficient and simple implementation. In the following, the structure of the SIR algorithm is presented.

\section{SIR Algorithm [15].}

1) For $i=1, \ldots, N$, a new particle $\chi_{k}^{i}$ is generated based on the pdf $p\left(\chi_{k} \mid \chi_{k-1}^{i}\right)$ and the corresponding weight is computed as $\rho_{k}^{i}=p\left(\psi_{k} \mid \chi_{k}^{i}\right)$.

2) Compute the sum of weights $\Omega_{w}=\sum_{i=1}^{N} \rho_{k}^{i}$ and then normalize the particle weights: $\rho_{k}^{i}=\Omega_{w}^{-1} \rho_{k}^{i}$.

3) Do a sampling process:

3.1. Start from $c_{0}=0$, construct the cumulative sum of weights (CSW) by computing $c_{i}=c_{i-1}+\rho_{k}^{i}$ for $i=1, \ldots, N$.

3.2. Give $i=1$ and generate a starting point $\tau_{1}$ from the uniform distribution $U\left[0, N^{-1}\right]$.

3.3. For $j=1, \ldots, N$

- Make $\tau_{j}=\tau_{1}+N^{-1}(j-1)$.

- While $\tau_{j}>c_{i}$ make $i=i+1$.

- Assign $\chi_{k}^{j}=\chi_{k}^{i}$.

- Assign $\rho_{k}^{j}=N^{-1}$ 


\section{B. Fault Detection and Isolation Based On Particle Filter}

The PF estimate output for the dynamic model described in (7) is:

$\hat{\psi}=\mathrm{C} \hat{\chi}$

where $\hat{\chi}$ is the PF state estimation outputs, which are determined as the output of the SIR algorithm applied for the system (7).

In fault diagnosis task, it is crucial significant to choose the effective residual, which can be used to easily distinguish between normal condition and fault condition when the system changing from a normal operation to a fault operation, and the corresponding threshold. In this paper, the error $\mathrm{e}$, which is defined as in (13), is chosen as the residual.

$$
\mathrm{e}=\psi-\hat{\psi}
$$

In normal operation, the Particle filter state tends to approximate the state variable of the system. Thus, from (7) and (12), the residual e tends to approximate the system uncertainties and noises, $\mathrm{e} \approx \vartheta+\Delta$, where $\vartheta \approx \omega+\zeta$.

Assumption 1: the system uncertainties and noises are bounded by $\|\vartheta+\Delta\| \leq e_{T h}$, where $e_{T h}$ is a known constant.

The assumption 1 is reasonable in real application because the noise value is usually bounded by a constant. In practice, the bound value of the system uncertainty and noise are usually obtained by experiments. Since e $\approx \vartheta+\Delta$ when the system in normal operation, the bound value of the uncertainty and noises, $\vartheta+\Delta$, can be estimated based on the bound value of the error e. In this paper, we employ this method. The procedure to obtain the bound value is performed offline, and is as follows. Firstly, a desired image is obtained by moving the robot to the target position and capturing the desired image. Then, starting from an arbitrary position, but guarantee that the object is placed within the FOV of camera, the robot is commanded to track the object using the control law (3). The fault diagnosis observer based on Particle filter is employed and the residual $\mathrm{e}$ is obtained when the system in normal operation. As shown in Figs. 4 and 12 (will be discussed latter), the residual e converges close to zero with small variation due to the noises and uncertainties. The bound value $e_{T h}$ is selected such that it is bigger than the peak value of the variation.

Robustness property: the robustness property of the fault detection scheme is to prevent a false alarm due to the effects of the system uncertainties and noises prior to the fault occurring. Since $\|e\| \approx\|\vartheta+\Delta\| \leq e_{T h}$ when the system in normal operation, by choosing $e_{T h}$ as the threshold, the robustness of the fault detection can be guaranteed. Fault decision is made when the residual, $(\|e\|)$, surpass its corresponding threshold $e_{T h}$.

From (1), the change of the state variables $u$ or $v$ can be represented by the change of $s$. Thus, in order to facilitate the process of fault detection and isolation of a feature point, the residuals of two state variables $u$ and $v$ should be represented by $s$, as follows:
TABLE I

FAULT-SIGNATURE TABLE

\begin{tabular}{|l|c|c|c|c|c|c|}
\hline Fault & $r_{1}$ & $r_{2}$ & $r_{3}$ & $r_{4}$ & $\ldots$ & $r_{n}$ \\
\hline None & 0 & 0 & 0 & 0 & $\ldots$ & 0 \\
\hline Sen. 1 & 1 & 0 & 0 & 0 & $\ldots$ & 0 \\
\hline Sen. 2 & 0 & 1 & 0 & 0 & $\ldots$ & 0 \\
\hline Sen. 3 & 0 & 0 & 1 & 0 & $\ldots$ & 0 \\
\hline Sen. 4 & 0 & 0 & 0 & 1 & $\ldots$ & 0 \\
\hline$\ldots$ & $\ldots$ & $\ldots$ & $\ldots$ & $\ldots$ & $\ldots$ & \\
\hline Sen. $\mathrm{n}$ & 0 & 0 & 0 & 0 & $\ldots$ & 1 \\
\hline
\end{tabular}

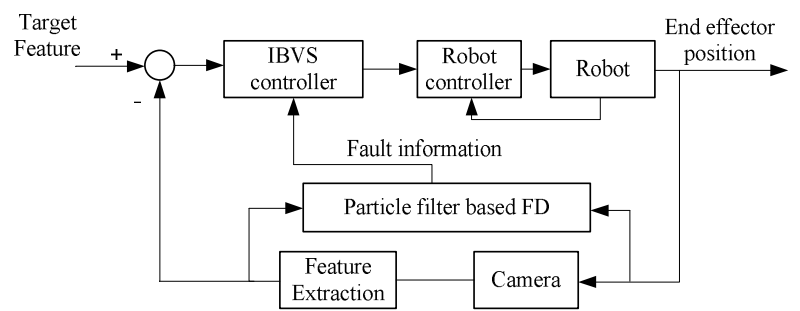

Fig. 2. Fault diagnosis and fault tolerant control scheme for visual servoing system.

$e_{s_{i}}=\sqrt{e_{u_{i}}^{2}+e_{v_{i}}^{2}}$

where $e_{u_{i}}$ and $e_{v_{i}}$ represent the PF estimation errors of the state variables $u_{i}$ and $v_{i}$ of the feature point $i$, respectively, and $e_{s_{i}}$ is used to represent the PF estimation error of the feature point $i$.

Then, the decision rule is defined as

$r_{i}= \begin{cases}0 & \text { if } e_{s_{i}} \leq T h_{i} \\ 1 & \text { if } e_{s_{i}}>T h_{i}\end{cases}$

where $T h_{i}=\sqrt{e_{T h_{u_{i}}}^{2}+e_{T h_{v_{i}}}^{2}}$, where $\|\vartheta+\Delta\|_{u} \leq e_{T h_{u}} \quad$ and $\|\vartheta+\Delta\|_{v} \leq e_{T h_{v}}$, is a chosen threshold.

The robustness property of the fault diagnosis system is guaranteed and can be explained as follows: when the system in normal operation, the residual is approximated as $e_{s_{i}} \approx \vartheta+\Delta$, and based on the assumption 1 , the residual is always smaller than the selected threshold value, i.e, $e_{s_{i}} \leq T h_{i}$ and $r_{i}=0$. However, when a fault occurs, the residual is approximated as $e_{s_{i}} \approx \vartheta+\Delta+\theta$. This residual signal will overshoots the threshold value $e_{s_{i}}>T h_{i}$ and $r_{i}=1$, the fault decision will be made. Fault detection and isolation rules are defined as in Table I.

In summary, fault detection system for a given visual servoing system is configured and calibrated based on the following steps:

Step 1: Identify the system uncertainties and noises, $\vartheta+\Delta$, by an offline experiment procedure explained above.

Step 2: Determine the bound value, $e_{T h}$, of the system uncertainty and noise. 


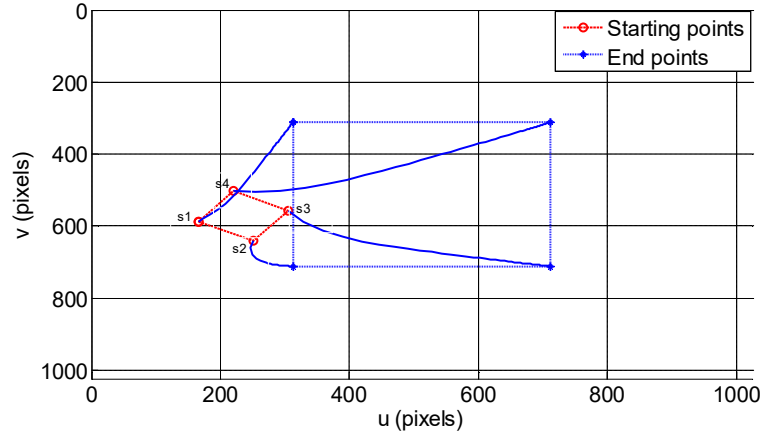

a)

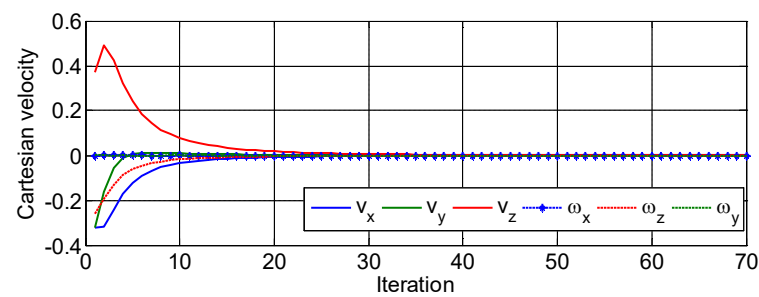

b)

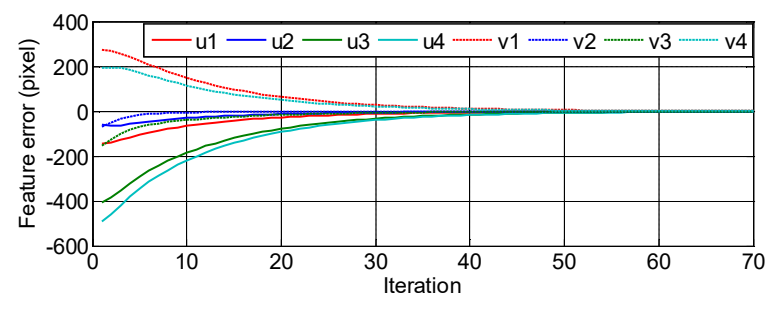

c)

Fig. 3. Tracking performance of simulated visual servoing when the system in normal operation. a) Image space, b) control inputs (noted that $\omega_{x} \equiv \omega_{y}$ in the figure), c) image error.

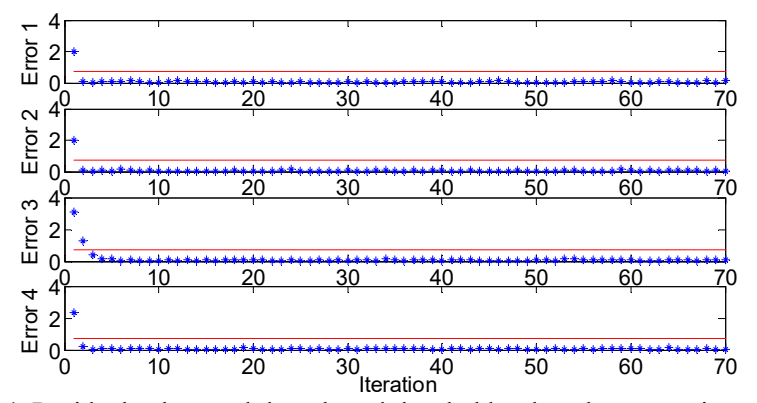

Fig. 4. Residual values and the selected thresholds when the system in normal operation.

Step 3: Set the bound value $e_{T h}$ as the threshold.

Step 4: Make a fault decision if the residual, $\|e\|$, surpass its corresponding threshold $e_{T h}$.

Remark 1: Table I is also used to define multiple faults working conditions. For example, when faults occur in the sensors 1,2 and 3 at the same time, the corresponding residuals are $r_{1}=1, r_{2}=1$ and $r_{3}=1$.

Remark 2: The threshold value is chosen as the same as the bound value of the system uncertainties and noises. Therefore, for a given visual servoing system, the threshold value should

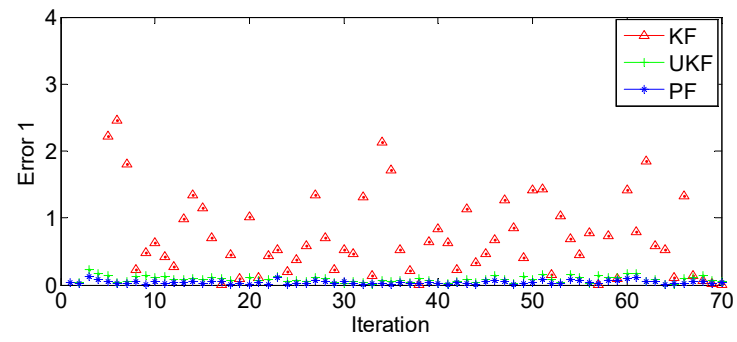

a) Error 1

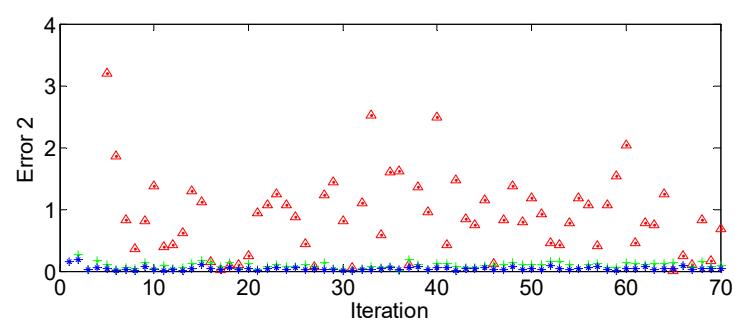

b) Error 2

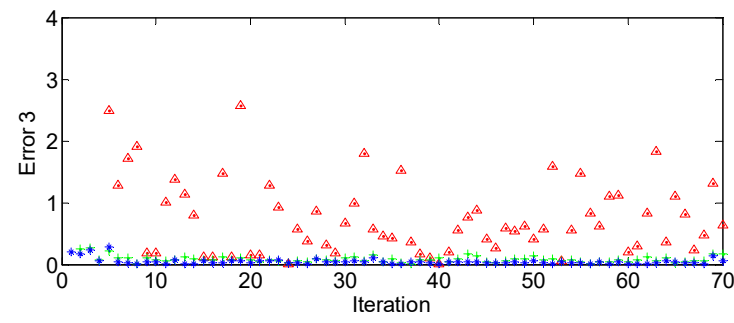

c) Error 3

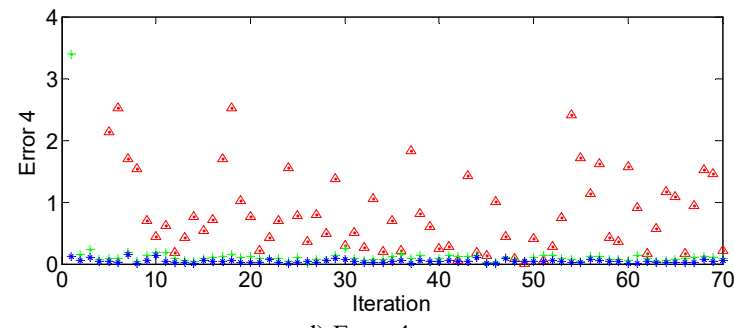

d) Error 4

Fig. 5. Comparison between Kalman filter (KF), unscented Kalman filter (UKF) and Particle filter (PF).

be calibrated based on the level of the system uncertainties and noises: the bigger the system uncertainties and noises, the bigger threshold need to be selected. However, it is necessary to obtain the precise bound value of the system uncertainties and noises prior to implementing the fault diagnosis system.

\section{C.Fault Estimation}

In the previous section, the analyses show that the PF approximates the image feature states with a very small error when the system in normal operation, $e_{s} \approx \vartheta+\Delta$. However, when a fault occurs at the time $T_{S}$, the estimation error $e_{S}$ tends to approximate the fault component, $e_{S} \approx \vartheta+\Delta+\theta$. Because $\vartheta+\Delta$ is usually much smaller compared to $\theta$, the estimation error approximates the fault magnitude, $e_{s} \approx \theta$. Thus, the fault magnitude can be approximated as 


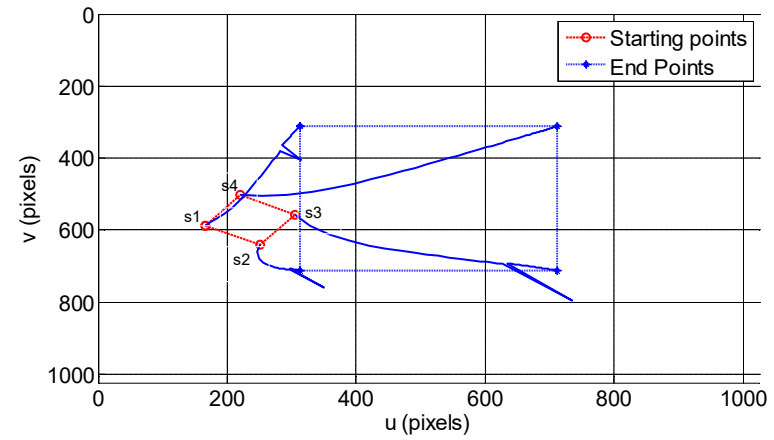

a)

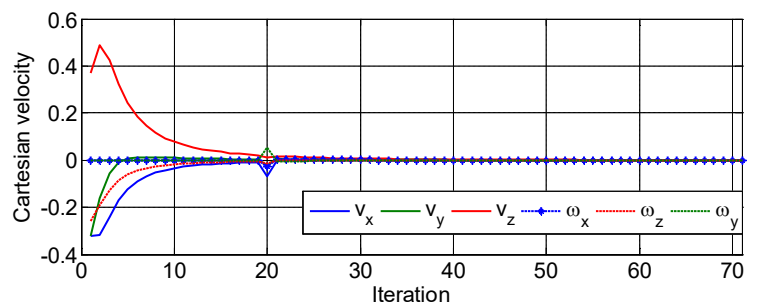

b)

Fig. 6. Tracking performance of simulated visual servoing when the faults in the points 1, 2 and 3 occur. a) Image space, b) control inputs.

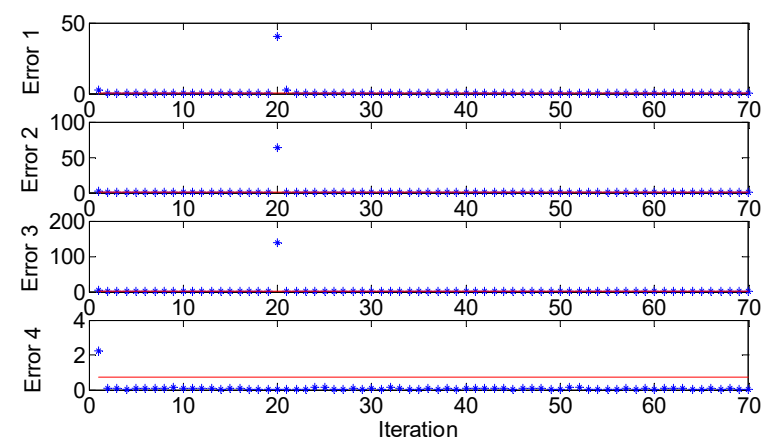

Fig. 7. Residual values when the faults in the points 1,2 and 3 occur.

$$
\Delta \mathrm{s}(t)_{t=T_{S}}=\mathrm{e}_{t=T_{S}}
$$

However, $T_{s}$ is an unknown time. It can only be predicted by using the information obtained from the fault detection and isolation scheme. Therefore, if we denote $T_{d}$ as the time when the fault is detected, fault magnitude can be estimated as

$\Delta \mathrm{s}(t)_{t=T_{s}}=\mathrm{e}_{t=T_{d}}$

where $\mathrm{e}_{t=T_{d}}$ denotes the PF estimation error at the time $T_{d}$. In practice, if the fault detection and isolation scheme works well, we will have $T_{d} \equiv T_{S}$.

\section{D.Fault Tolerant Control}

After a fault is diagnosed, it is desired that the fault should be compensated to reduce its effects in the system. In the traditional visual servoing, the system is controlled by the conventional law (3). The desired system performance is satisfied only when it operates in normal condition, wherein the system gets the feedback from the correct image feature input $s$. However, when a fault occurs, the fault feature value $\bar{s}(t)=s(t)+\Delta s(t)$ is used as the input signal to the controller
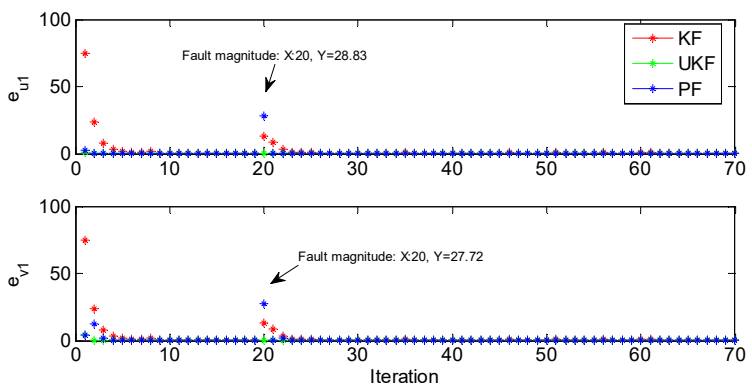

a)
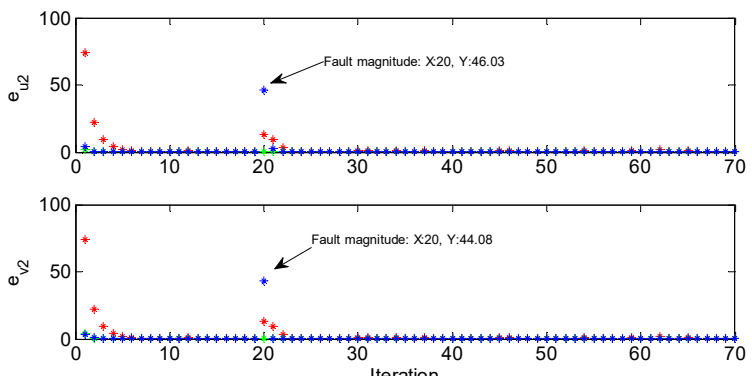

b)
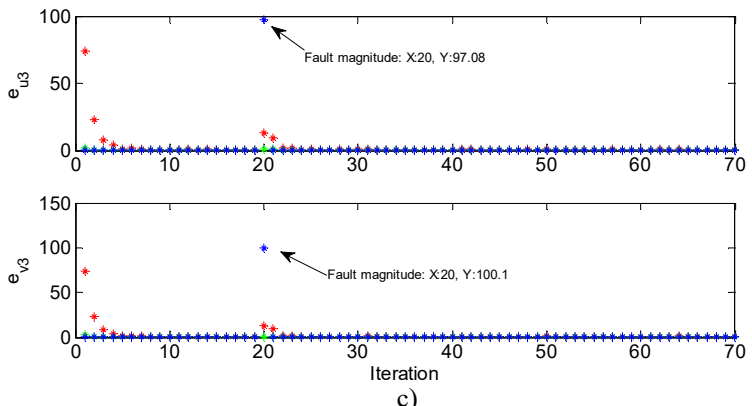

Fig. 8. KF, UKF and PF state estimation errors when the faults in the points 1 , 2 and 3 occur. a) KF, UKF and PF estimation errors for the feature point $1, b$ ) $\mathrm{KF}$, UKF and PF estimation errors for the feature point 2, and c) KF, UKF and $\mathrm{PF}$ estimation errors for the feature point 3.

that will generate incorrect control input and decrease the tracking performance consequently. In order to increase the system performance, the correct image feature value $s(t)$ at the time $T_{S}$ should be reconstructed and fed back to the controller instead of the fault value $\bar{s}(t)$. The correct feature value can be simply calculated as $s(t)=\bar{s}(t)-\Delta s(t)$. However, since the correct fault value $\Delta s(t)$ cannot be calculated, its estimation value, which is obtained from the fault estimation scheme (17), is used instead. Then, the value of the correct image feature at the time $T_{s}, \hat{s}(t)$, can be estimated as:

$\hat{s}(t)=\bar{s}(t)-\mathrm{e}_{t=T_{d}}$

Afterward, when a fault is detected, to reduce its effect on the system, the controller is reconfigured as

$V_{c}=-\lambda \hat{\mathrm{L}}_{\mathrm{s}}^{+}\left(\bar{s}(t)-\mathrm{e}_{t=T_{d}}-\mathrm{s}^{*}\right)$

Finally, the whole FTC law for the visual servoing system is designed as: 
TABLE II

COMPARISON BETWEEN KALMAN FILTER, UNSCENTED KALMAN FILTER (UKF) AND PARTICLE FILTERS (PFS)

\begin{tabular}{|c|c|c|c|c|c|c|c|c|c|c|}
\hline \multirow{2}{*}{\multicolumn{2}{|c|}{ Method }} & \multicolumn{2}{|c|}{ Point 1} & \multicolumn{2}{|c|}{ Point 2} & \multicolumn{2}{|c|}{ Point 3} & \multicolumn{2}{|c|}{ Point 4} & \multirow{2}{*}{$\begin{array}{c}\text { Computation } \\
\text { time }\end{array}$} \\
\hline & & RMSE & VAR & RMSE & VAR & RMSE & VAR & RMSE & VAR & \\
\hline & $\mathrm{KF}$ & 0.6649 & 0.5343 & 0.8436 & 0.6272 & 0.8348 & 0.6554 & 0.7548 & 0.5801 & 0.000168 \\
\hline & UKF & 0.0903 & 0.0420 & 0.0892 & 0.0425 & 0.0896 & 0.0515 & 0.0971 & 0.0438 & 0.000754 \\
\hline $\mathrm{P}$ & $\mathrm{N}=200$ & 0.0922 & 0.0595 & 0.1065 & 0.1061 & 0.0995 & 0.0528 & 0.1072 & 0.1250 & 0.009644 \\
\hline $\mathrm{F}$ & $\mathrm{N}=500$ & 0.0450 & 0.0256 & 0.0478 & 0.0250 & 0.0450 & 0.0312 & 0.0496 & 0.0289 & 0.019350 \\
\hline
\end{tabular}

$V_{c}= \begin{cases}-\lambda \hat{\mathrm{L}}_{\mathrm{S}}^{+}\left(s-s^{*}\right) & t \neq T_{d} \\ -\lambda \hat{\mathrm{L}}_{\mathrm{S}}^{+}\left(\bar{s}(t)-\mathrm{e}-s^{*}\right) & t=T_{d}\end{cases}$

The overall FD and FTC schemes developed in this paper are illustrated in Fig. 2.

\section{SimULATION STUDY}

In this section, the performances of the visual servoing system with and without FTC are simulated to show the effectiveness of the FD and FTC schemes. The target used in this simulation is masked by four feature points. The image resolution is $1000 \times 1000$ pixel. The sampling time is 5 frameper-second (fps). The number of particles is set as $N=500$; this value is chosen based on the trial and error validation through several experiments. Eight internal states, $u_{1}, v_{1}, u_{2}, v_{2}, u_{3}, v_{3}$ and $u_{4}, v_{4}$ are approximated by the PF. Starting from the initial camera location, where the four points can be seen by dashed lines in the image space in Fig. 3a), the target of the visual servoing system is to locate the camera at the position such that the four points can be seen by dot-dashed lines in the image space in Fig. 3a). In order to compare the tracking performance of the system among normal operation, fault operation without FTC and fault operation with FTC, the visual servoing system is modeled in three different working conditions. In the first case, the visual servoing system is modeled to operate in normal condition. In the second case, a multiple faults condition is generated to the system without FTC to illustrate both the single and multiple faults effects. In the third case, the proposed FTC control law is employed to reduce the effects of faults generated in the second case.

\section{A. Visual Servoing System in Normal Operation}

Considering the operation of the system in normal operation, as shown in Fig. 3, the PF approximates the nonlinear visual servoing system with a small error due to the uncertainties and noise, $\vartheta+\Delta$, as shown in Fig. 4 . We can see from Fig. 3 that the camera tracks the object very well. From Fig. 4, the PF estimation errors converge close to zero very quick (after a few iterations). To distinguish between the effects of the uncertainties and faults, the threshold values $T h$ are selected as the red line, as shown in Fig. 4.

To further evaluate the performance of the PF to approximate the system states, we simulate the system with the measurement noise $\delta=5$. In addition, we compare the performance of the $\mathrm{PF}$ with different number of particles used with Kalman filter (KF) and unscented Kalman filter (UKF).

The approximation errors of KF, UKF and PFs are shown in Fig. 5. For easy in comparison, the root mean square error

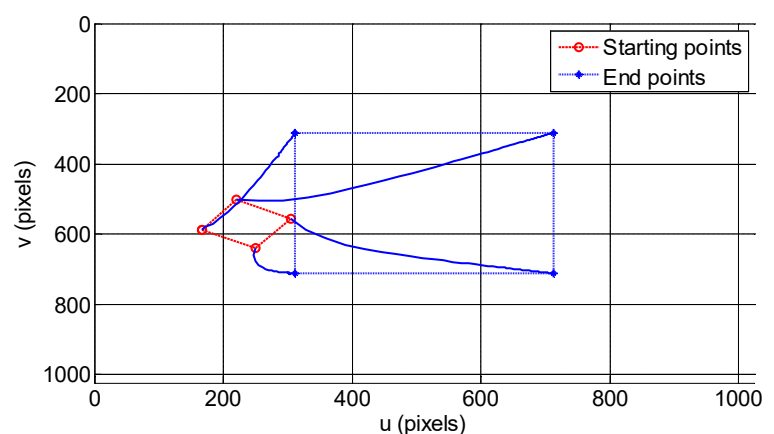

a)

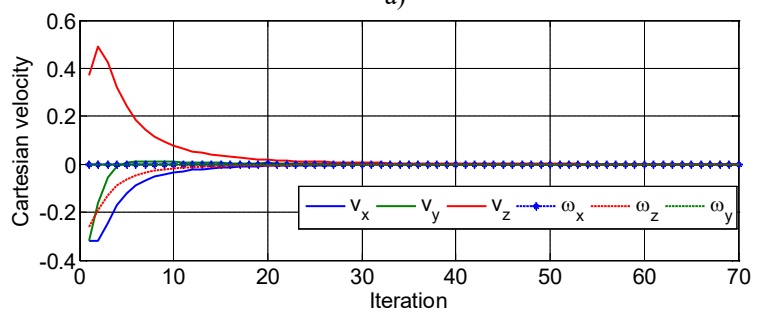

b)

Fig. 9. Tracking performance of simulated visual servoing when the faults existed in feature points 1,2 and 3 with FTC. a) Image space, b) control inputs (noted that $\omega_{x} \equiv \omega_{y}$ in the figure).

(RMSE) and standard deviation (STD) and the computation time of these methods are also reported in Table II. From the results we can see that the PF filters provide a lower RMSE and STD than the KF and UKF. On the other hand, the used of higher number of particle provides a better performance: the performance of the PF with $\mathrm{N}=500$ is better than the PF with the lower number particles $(\mathrm{N}=200)$. However, there is a tradeoff between the approximation capability and the computation time of the PF. The higher number of particles the better approximation performance but higher computation time, and vice versa. Since the PF generated the residual smaller than KF and UKF, the threshold for the system using $\mathrm{PF}$ can be set as a smaller value compared to the use of KF or UKF. The lower threshold value has several advantages such as reducing the detection time and increasing the sensitivity of the fault detection system [23]. However, according to the Table I, the computation time of the PFs is much higher compared to the KF and UKF. Fortunately, this still guarantees the real time computation of visual servoing system.

\section{B. Visual Servoing System with Assumed Virtual Sensor Faults}

In order to show the effects of virtual sensor bias fault in the visual servoing system and to verify the performance of the developed FD and FTC schemes, we generate a bias fault to the visual servoing system. Particularly, we simulate the 
system with a multiple faults condition, $\Delta s_{1}=[30,30]$, $\Delta s_{2}=[50,50]$ and $\Delta s_{3}=[100,100]$. The three faults are assumed to be occurred at the same time at the iteration 20 . Figure 6 illustrates the variation of the system performance when the system changes from normal operation to fault operation. Comparison results between Fig. 3 and Fig. 6 show that the motion of the camera is incorrect when the displacement of a feature is incorrectly extracted. In particular, due to the effect of the virtual sensor faults, the corresponding velocity control input is discontinuous at the iteration 20 , as shown in Fig. 6b), that will make the visual servoing system unstable. Due to the effect of the fault, the convergence of the $\mathrm{PF}$ is broken, as shown in Fig. 7. The residuals of the feature points 1, 2 and 3 overshoot the corresponding thresholds at the iteration 20, indicating that the faults are existed in the virtual sensors 1, 2 and 3 . Thus, in this experiment, the system has detected and isolated the faults successfully.

In the next, we consider fault estimation performance. At the iteration 20 , where the existing faults in the feature points 1,2 and 3 were detected and isolated successfully, fault estimations were then calculated by using the formulation defined in (17). Fig. 8 shows the fault estimation results using the KF, UKF and PF. According to (17) and Fig. 8, the computed fault estimations using PF are $\hat{\Delta} s_{1}=\mathrm{e}_{t=T_{d}=20}=[28.83,27.72]$ for the virtual sensor $1, \hat{\Delta} s_{2}=\mathrm{e}_{t=T_{d}=20}=[46.03,44.08]$ for the virtual sensor 2, and $\hat{\Delta} s_{3}=\mathrm{e}_{t=T_{d}=20}=[97.08,100.1]$ for the virtual sensor 3 . The estimated fault values, $\hat{\Delta} s_{1}, \hat{\Delta} s_{2}$ and $\hat{\Delta} s_{3}$, are very close to the assumed fault values, $\Delta s_{1}, \Delta s_{2}$ and $\Delta s_{3}$. In addition, the comparison results shown in Fig. 8 verify that the PF provides better fault estimation compared to the KF and UKF. Thus, from the comparison results shown in Fig. 5 and Fig. 8, we can verify that the PF gives higher performance than the KF and UKF for both fault detection and estimation.

\section{Visual Servoing System With Fault Tolerant Control}

As shown in Fig. 5b), the effects of the faults generate a discontinuous control input, and consequently break the stability of the system. To reduce the effects of the faults, the developed FTC law in (20) is employed based on the feedback information of the estimated fault using PF obtained in Fig. 8. The performance of the developed FTC for the visual servoing is shown in Fig. 9. Comparison results between Fig. 9 and Figs. 2 and 5 show that the FTC system compensates the effects of the faults very well. The system performance of the visual servoing under FTC is comparable to the normal operation. The velocity control input of the system with FTC is continuous, as shown in Fig. 9b). Thus, we can conclude that the fault has been accommodated successfully.

Remark 3: If we consider the sensor fault only and assume that the actuator (camera motion) is always healthy, the proposed fault diagnosis and accommodation can detect and compensate for the heavy sensor fault case, where all the sensors are failed at the same time. However, if we consider

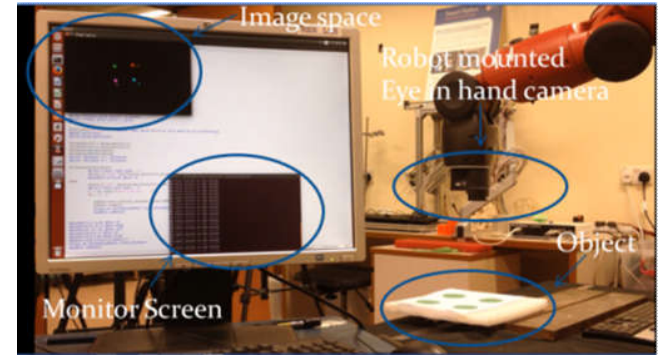

Fig. 10. Experiment setup of eye-in-hand visual servoing.

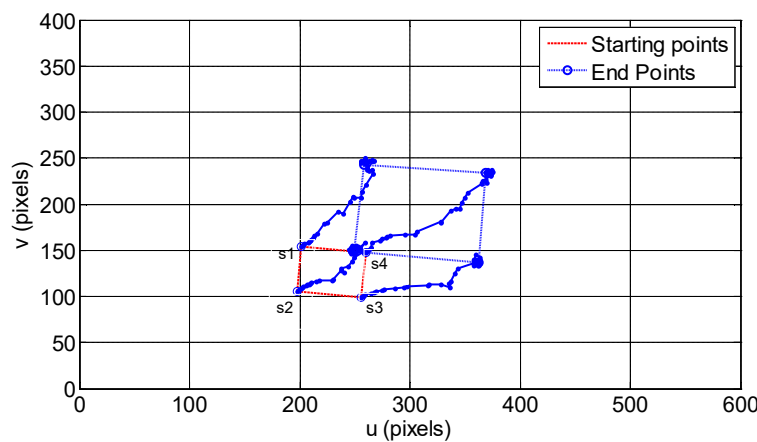

a)

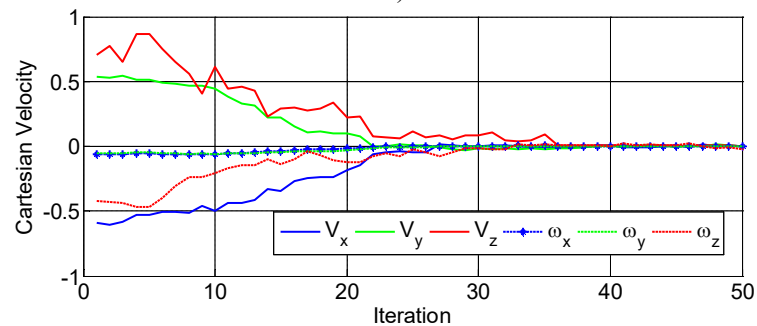

b)

Fig. 11. Tracking performance of experimented visual servoing when the system in normal operation. a) Image space, b) control inputs.

both the actuator and sensor fault scenarios, the control system will isolate a wrong actuator fault instead of sensor faults if all the sensors are failed at the same time [12]. Fortunately, this situation is rarely occurring in real applications.

Remark 4: There are many active fault tolerant approaches published in the literature [21]. Among them, the approach, which uses a nominal controller plus a fault compensator, which is taken from a fault estimation scheme, is mostly applied because it is simple in design and effective in fault compensation [23, 24]. This paper follows this design procedure. It is obvious to see that the performance of this FTC strategy is mainly dependent on the performance of the fault estimation. Therefore, instead of comparing the performance among AFTC approaches, we compare the performance of fault estimation using KF, UKF and PF. The results shown that the $\mathrm{PF}$ provides very accurate fault estimation, and thus the developed AFTC would be effective compared to other FTC approaches.

\section{EXPERIMENTAL STUDY}

In order to show the tracking performance of the system with FD and FTC, a lab experimental setup is developed as 

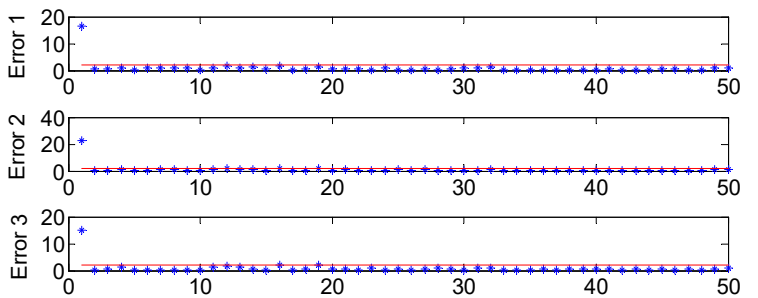

\begin{tabular}{ll|l|l|l|}
\hline & 40 & & & \\
\hline
\end{tabular}

Fig. 12. Residual values of experimented visual servoing and the selected threshold values.

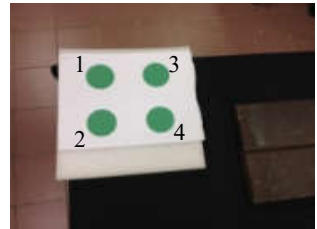

a)

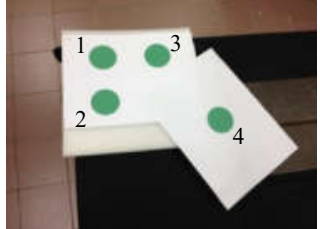

b)
Fig. 13. Image feature in a) normal extraction, b) virtual sensor bias fault.

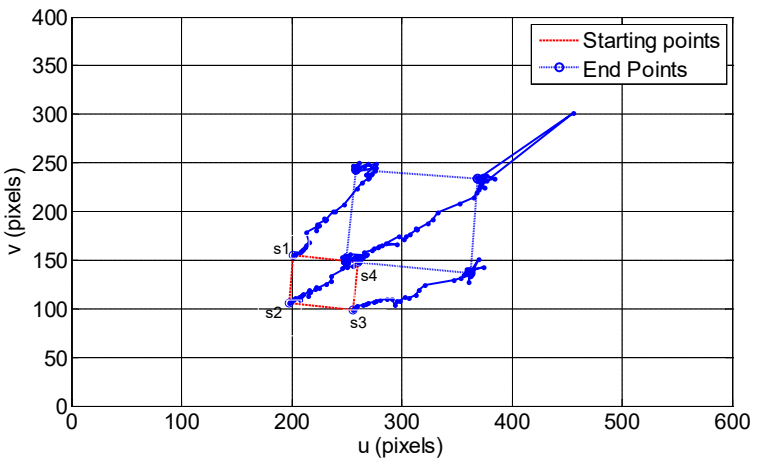

a)

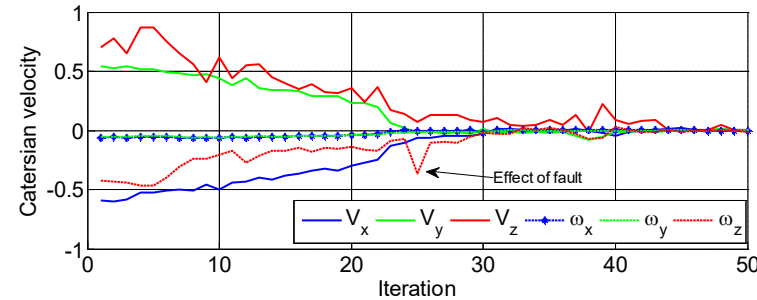

b)

Fig. 14. Tracking performance of visual servoing when the fault existed in the virtual sensor (feature point 4) without FTC. a) Image space, b) control inputs.

shown in Fig. 10. The Baxter industrial robot [25] is used to perform experiment. The Baxter is a new generation industrial robot and has been widely using in industrial application and research. The Baxter has two independent arms and each has seven degree-of-freedom (DOF). Each arm was attached with an eye-in-hand configuration. In this study, we used the leftarm and left-hand camera to do experiments. The object to be tracked includes four feature points, as shown in Fig. 13. The camera capturing rate is $30 \mathrm{fps}$ (frame/s), and the image information is sent into the host Linux PC to processing. The camera of the Baxter robot has $640 \times 400$ pixels resolution and has an effective focal length of $1.2 \mathrm{~mm}$.
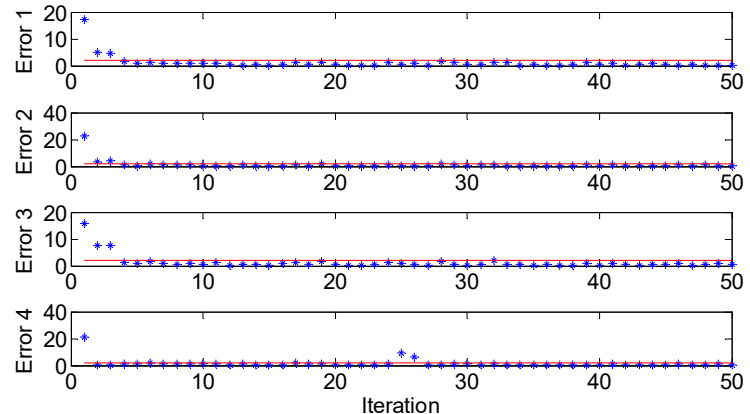

Fig. 15. PF estimation errors when the experimented visual servoing sytem in normal operation.

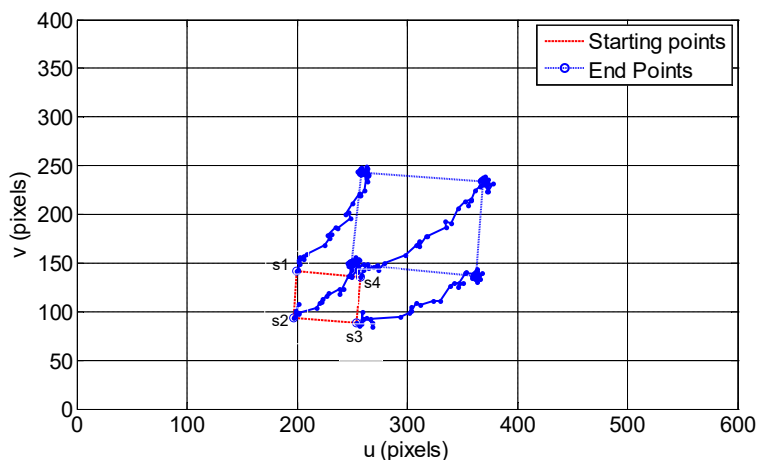

a)

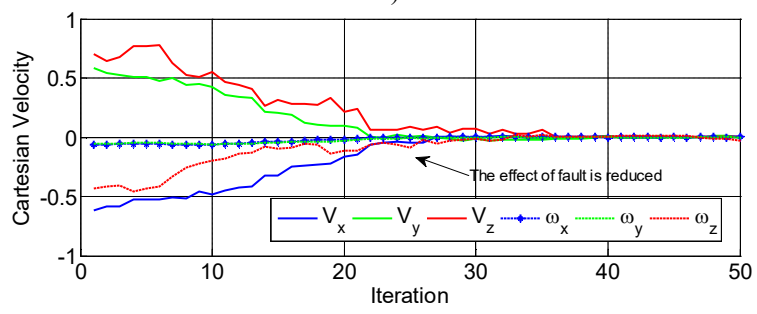

b)

Fig. 16. Tracking performance of the experimented visual servoing system when the fault existed in the feature point 4 with FTC. a) Image space, b) control inputs.

Faults are introduced in the virtual sensor by changing the displacements of the image feature points at an arbitrary time. In the following, we present the performance of the visual servoing system without FTC and with FTC when the system in normal and fault operations.

\section{A. Visual Servoing in Normal Operation}

In fault-free working condition, the visual servoing system tracks the object very well, as shown in Fig. 11. The PF estimation errors, which are used as the residuals in this paper, are shown in Fig. 12. The results from Fig. 12 show that the PF estimation errors are quickly convergent after a few iterations. As analyzed in section IIIB, the residual value obtained when the system in fault-free operation is the uncertainty and noise components, $\vartheta+\Delta$, of the system. Thus, to avoid any incorrect fault decision due to the effects of the system uncertainties and noises, the threshold values $T h$ are selected to be bigger than the bound value of $\vartheta+\Delta$. The selected thresholds are the red lines shown in Fig. 12. 


\section{B. Visual Servoing System under Virtual Sensor Bias Fault without Fault Tolerant Control}

In the presence of a bias sensor fault, the controller system read a noise feature value instead of the true designed feature, as an example shown in Fig. 1. To simulate a bias fault, we change the displacement of the feature point 4 at the iteration 25, as illustrated in Fig. 13. Fig. 14 shows the transition of the tracking performance when changing from normal operation to fault operation. From Fig. 14b), due to the presence of the fault at the iteration 25 , the computed control velocity input is changed suddenly. Due to the effects of the fault, the convergence of the PF estimation error is broken, as shown in Fig. 15. In Fig. 15, the residual of the feature point 4 overshoots the corresponding threshold at the iteration 25, and thus the fault decision is made.

\section{Visual Servoing with Fault Tolerant Control Under Virtual Sensor Fault}

The generated bias virtual sensor fault provides discontinuous control input as shown in Fig. 14b). To tackle this problem, the developed FTC law in (20) is employed. Fig. 16 shows the results of the fault compensation. By comparing Fig. 16b) with Fig. 14b), we can see that the effects of the fault in the computed control input are much reduced. From this, we can conclude that the fault has been estimated precisely and its effects in the visual servoing system have been correctly compensated by the developed FTC law.

Remark 5: The performance of the fault estimation and accommodation is dependent on the level of uncertainty and noise of the visual servoing system.

\section{CONCLUSION}

This paper reviews the failure scenarios of the feature extraction task in visual servoing system, namely virtual sensor bias fault. Then, the PF-based FD is developed to monitor the presence of the faults. An AFTC is developed based on the estimated fault information to compensate for the effects of the faults. Both the designed FD and FTC schemes have a simple structure and easy implementation in real application. Simulation and experimental results verify that the presence of the failures due to the sensor bias faults can be detected accurately and its effects can be compensated effectively.

According to [12], the failure of visual servoing system could be caused by incorrect robot motion, namely actuator fault. Fault estimation and accommodation for the failures of actuator fault will be investigated in our future work.

\section{REFERENCES}

[1] M. Keshmiri, W. F. Xie, and A. Mohebbi, "Augmented image-based visual servoing of a manipulator using acceleration command," IEEE Trans. Ind. Electron., vol. 61, no. 10, pp. 5444-5452, 2014.

[2] D. Ceglarek, M. Colledani, J. Vancza, D. Y. Kim, C. Marine, M. KogelHollacher, A. Mistry, L. Bolognese, "Rapid Deployment of Remote Laser Welding Processes in Automotive Assembly Systems," Annals of the CIRP, vol. 64, no. 1, pp. 389-394, 2015.

[3] Y. Ding, P. Kim, D. Ceglarek, J. Jin, "Optimal Sensor Distribution for Variation Diagnosis in Multi-station Manufacturing Processes," IEEE Trans. Robot. Auto., vol. 19, no. 4, pp. 543-556, 2003.
[4] A. Hajiloo, M. Keshmini, W. F. Xie, T. T. Wang, "Robust online model predictive control for a constrained image based visual servoing," IEEE Trans. Ind. Electron., vol. 63, no. 4, pp. 2242-2250, 2016.

[5] W. F. Xie, Z. Li, X. W. Tu, C. Perron, "Switching control of imagebased visual servoing with laser pointer in robotic manufacturing systems," IEEE Trans. Ind. Electron., vol. 56, no. 2, pp. 520-529, 2009.

[6] P. Corke, F. Spindler, F. Chaumetter, "Combining Cartesian and polar coordinates in IBVS," in Proc. IEEE/RSJ Int. Conf. Intell. Robots Syst., (IROS'09), Oct. 2009, pp. 5962-5967.

[7] J. Wang, H. Cho, "Micropeg and hole alignment using image moments based visual servoing method," IEEE Trans. Ind. Electron., vol. 53, no. 3, pp. 1286-1294, 2008.

[8] N. G. Aracil, E. Malis, R. A. Santonja, C. P. Vidal, "Continuous visual servoing despite the changes of visibility in image features," IEEE Trans. Robot., vol. 20, no. 4, pp. 713-723, 2004.

[9] G. Chesi, K. Hashimoto, D. Prattichizzo, and A. Vicino, "Keeping features in the field of view in Eye-In-Hand visual servoing: A switch approach," IEEE Trans. Robot., vol. 20, no. 5, pp. 908-913, 2004.

[10] M. Kazemi, K. K. Gupta, M. Mehrandezh, "Randomized kinodynamic planning for robust visual servoing," IEEE Trans. Robot., vol. 29, no. 5, pp. 195-200, 2010.

[11] M. Baumann, S. Leonard, E. A. Croft, J. J. Little, "Path planning for improved visibility using a probabilistic road map," IEEE Trans. Robot., vol. 26, no. 1, pp. 1197-1211, 2013.

[12] M. Van, D. Wu, S. S. Ge, H. Ren, "Fault Diagnosis in Image-Based Visual Servoing with Eye-in-Hand Configuration Using Kalman Filter," IEEE Trans. Ind. Inform., vol. 12, no. 6, pp. 1998-2007, 2016.

[13] L. C. B. Preciado, O. Y. Sergiyenko, J. C. R. Quinonex, X. Garcia, V. V. Tyrase, M. R. Lopez, D. H. Balbuena, P. Mercorelli, M. Podrygalo, A. Gurko, I. Tabakova, O. Starostenko, "Optical 3D laser measurement system for navigation of autonomous mobile robot," Optics and Lasers in Engineering, vol. 54, pp. 159-169, 2014.

[14] M. Van, D. Wu, S. S. Ge, H. Ren, "Condition monitoring for image based visual servoing using Kalman Filter," Advances in Visual Computing, pp. 842-850, 2015.

[15] M. S. Arulampalam, S. Maskell, N. Gordon, ans T. Clapp, "A tutorial on particle filters for online nonlinear/non-Gaussian Bayesian tracking," IEEE Trans. Signal Process., vol. 50, no. 12, pp. 174-188, 2012.

[16] T. Wei, Y. Huang, C. L. Philip Chen, "Adaptive sensor fault detection and identification using particle filter algorithms," IEEE Trans. Syst. Man Cyber., vol. 39, no. 2, pp. 201-213, 2009.

[17] N. Widynski, M. Mignotte, "A multiscale particle filter framework for contour detection," IEEE Trans. Pattern. Analy. Machine. Intel., vol. 36, no. 10, pp. 1922-1935, 2014.

[18] B. Zhao, R. Skjetne, M. Blanke, and F. Dukan, "Particle filter for fault diagnosis and robust navigation of underwater robot," IEEE Trans. Control Syst. Tech., vol. 22, no. 6, pp. 2399-2407, 2014.

[19] S. Laghrouche, J. Liu, F. S. Ahmed, M. Harmouche and M. Wack, "Adaptive Second-Order Sliding Mode Observer-Based Fault Reconstruction for PEM Fuel Cell Air-Feed System," IEEE Trans. Control Syst. Tech., vol. 23, no. 3, pp. 1098-1109, 2015.

[20] J. Liu, W. Luo, X. Yang and L. Wu, "Active fault tolerant control systems," IEEE Trans. Ind. Electron., vol. 63, no. 5, pp. 3261-3270, 2016.

[21] M. Mahmoud, J. Jiang, Y. Zhang, Active fault tolerant control systems: stochastic analysis and synthesis, Lecture Notes in Control and Information Sciences, Springer Sicence \& Business Media, vol. 287, 2003.

[22] M. Van, S. S. Ge, H. Ren, "Robust Fault-Tolerant Control for a Class of Second-Order Nonlinear Systems Using an Adaptive Third-Order Sliding Mode Control," IEEE Trans. Systems, Man, and Cybern.: Systems , vol. 47, no. 2, pp. 221-228, 2017.

[23] M. Van, H. J. Kang, Y. S. Suh, K. S. Shin, "A robust fault diagnosis and accommodation scheme for robot manipulators," Int. J. Control Aut. Syst., vol. 11, no. 2, pp. 377-388, 2013.

[24] M. Van, S. S. Ge, H. Ren, "Finite Time Fault Tolerant Control for Robot Manipulators Using Time Delay Estimation and Continuous Nonsingular Fast Terminal Sliding Mode Control," IEEE Trans. Cybern., $\quad$ vol.PP, no.99, pp.1-13, doi: 10.1109/TCYB.2016.2555307, 2016.

[25] C. Fitzgerald, "Developing baxter," in Proc. Int. Conf. technol. Practical Robot Appl., Woburn MA, USA, 2013, pp. 1-6. 


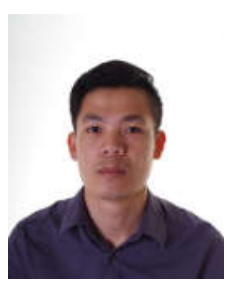

Mien Van received his B.S. degree in Electrical Engineering from Danang University of Technology, DaNang city, Vietnam, in 2009. He received his Ph.D. degree in the School of Electrical Engineering, University of Ulsan, South Korea in 2015.

He was a Post-Doctoral Research Fellow with the Advanced Robotics Centre, Faculty of Engineering, National University of Singapore, Singapore and the WMG, University of Warwick, UK, from 2015. He is currently with the School of Science and Technology, Nottingham Trent University, UK. His research interests include Assistive robotics, Robot control, Robot-vision system, Fault diagnosis and fault tolerant, Machine learning, and Sensing and Perception.

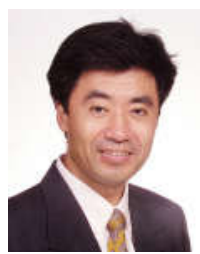

Shuzhi Sam Ge (S'90-M'92-SM'00-F'06) received the B.Sc. degree from the Beijing University of Aeronautics and Astronautics, Beijing, China, in 1986, and the Ph.D. degree from the Imperial College of Science, Technology and Medicine, University of London, London, U.K., in 1993.

$\mathrm{He}$ is the Director of Social Robotics Laboratory, Smart Systems Institute, and the Professor with the Department of Electrical and Computer Engineering, National University of Singapore, Singapore. He has co-authored seven books and over 300 international journal and conference papers. His current research interests include social robotics, adaptive control, intelligent systems, and artificial intelligence. Prof. Ge is an Editor-in-Chief of the International Journal of Social Robotics, Springer. He has served/been serving as an Associate Editor for a number of flagship journals, including the IEEE TRANSACTIONS ON AUTOMATIC CONTROL, the IEEE TRANSACTIONS ON CONTROL SYSTEMS TECHNOLOGY, the IEEE TRANSACTIONS ON NEURAL NETWORKS, and Automatica. He also serves as a Book Editor of the Taylor \& Francis Automation and Control Engineering Series. At the IEEE Control Systems Society, he served/serves as the Vice President for Technical Activities, from 2009 to 2010, the Vice President of Membership Activities, from 2011 to 2012, a Member of Board of Governors of the IEEE Control Systems Society, from 2007 to 2009. He is a fellow of the International Federation of Automatic Control, the Institute of Engineering and Technology, and Singapore Academy of Engineering.

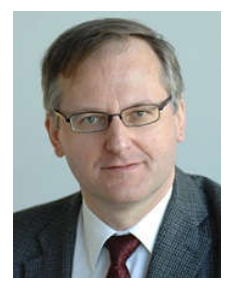

Dariusz Ceglarek (SM'13) is EPSRC Star Research Chair at WMG, University of Warwick and a CIRP Fellow. Previously, he was Professor in Industrial and Systems Engineering at University of Wisconsin, Madison. He received his Ph.D. in Mechanical Engineering from University of Michigan-Ann Arbor in 1994. His research focusses on digital manufacturing, inprocess quality control and root cause analysis across design, manufacturing and service. His research has been funded by: US (NSF, NIST), United Kingdom (EPSRC, InnovateUK and HVM Catapult) and EU (FP7, Marie Curie) and industry (more than 30 OEMs and SMEs). He has published over 150 papers and received several Best Paper Awards. He has received numerous awards including 2007 UK EPSRC Star Award, US NSF 2003 CAREER Award; 1999 Outstanding Research Scientist Award from University of Michigan; the 1998 Dell K. Allen Outstanding Young Manufacturing Engineer of the Year Award from the SME. He has served on numerous Editorial Boards and is an Associate Editor (Europe) of the ASTM Smart and Sustainable Manufacturing Systems Journal. Prof. Ceglarek served as Chair of the Quality, Statistics and Reliability Section of INFORMS; Program Chair for the ASME Design-for-Manufacturing Life Cycle Conferences, Assoc Editor of the IEEE Trans., and of the ASME Trans, J. Manuf Sys \& Eng. 